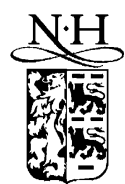

ELSEVIER

\title{
Breathing domain wall mode of an interface pinned Nèel wall
}

\author{
A.L. Dantas ${ }^{\text {a }}$, M.S. Vasconcelos ${ }^{\mathrm{a}}$, A.S. Carriço ${ }^{\mathrm{b}, *}$ \\ a Departamento de Física, Universidade do Estado do Rio Grande do Norte, 59.610-210 - Mossoró-RN, Brazil \\ ${ }^{\mathrm{b}}$ Departamento de Física, Teórica e Experimental, Universidade Federal do Rio Grande do Norte, 59.072-970 - Natal-RN, Brazil
}

\begin{abstract}
A step defect at the interface between a two - sublattice antiferromagnetic substrate and a uniaxial ferromagnetic thin film stabilizes a Nèel wall. Breathing domain wall modes (BDWM) are studied with the external field in the plane of the film. The restoring force of the domain wall excitations originates from the interface exchange energy within the domain wall. For low anisotropy ferromagnets there is a significant reduction of the domain wall width and the frequency of the domain wall excitations is determined from the interface exchange coupling in a small interface area. For strong interface coupling, the width of the domain wall excitation spectrum scales with the square root of the ratio between the interface effective exchange field and the anisotropy field of the ferromagnetic film. (C) 2001 Elsevier Science B.V. All rights reserved.
\end{abstract}

Keywords: Domain wall excitations; Domain wall pinning; Interface effects

Magnetic force microscopy (MFM) is a powerful technique to map the magnetic structure of materials. Amongst the possible excitations of domain walls the breathing domain wall mode is most explored since the stray field produced by the breathing magnetic charge is large in the neighborhood of the magnetic film surface where the domain wall center is located. Thus, the study of breathing domain wall modes by MFM is a good method for mapping the positions of the domain walls and thus the sizes and orientation of the domains [1]. This might be of interest for the characterization of defects in interfaces between a ferromagnetic $(F)$ film and an antiferromagnetic (AF) substrate. Despite the large amount of research dedicated to the study of the magnetic properties of $\mathrm{F} / \mathrm{AF}$ interfaces, there are still unsolved questions [2].

Interface roughness in bilayers consisting of a thin ferromagnetic (F) film on an antiferromagnetic (AF) substrate may lead to the nucleation of domain walls $[3,4]$. Domain wall nucleation has also been observed in the vicinal $\mathrm{F} / \mathrm{AF}$ interfaces [5-8]. In these cases, the interface

\footnotetext{
* Corresponding author.

E-mail address: acarrico@dfte.ufrn.br (A.S. Carriço).
}

pattern is controlled to a reasonable degree of accuracy. In the experiments on $\mathrm{Fe} / \mathrm{Cr} / \mathrm{Fe}$ trilayers with a wedge $\mathrm{Cr}$ spacer $[5,6]$, the periodic domain structure in the thinner Fe layer was found to correspond to the $\mathrm{Cr}$ interface steps induced by the vicinal orientation of one of the $\mathrm{Cr}$ surfaces of the wedge.

Rigid displacement domain wall modes [9] as well as local spin wave modes [10] have been recently studied in Nèel walls pinned by interface step defects. One key aspect of this system is that the anisotropy field of many ferromagnets of current interest is much smaller than a typical exchange field. Therefore, it is likely that the anisotropy field of the ferromagnet is small (by two or three orders of magnitude) compared to the interface exchange field in a $\mathrm{F} / \mathrm{AF}$ bilayer. Thus, the interface coupling may lead to a considerable reduction in the domain wall width [11].

The magnetic energy fluctuations induced by variations in the domain wall width are proportional to the inverse of the domain wall width, as shown below. Thus, the measurement of breathing domain wall excitations, for domain walls pinned at $\mathrm{F} / \mathrm{AF}$ step defects, might be an efficient means of estimating the effective interface exchange field. 
For a single-step defect the magnetic energy density is given by

$$
\begin{aligned}
E= & \int_{-L}^{L}\left[A \theta_{y}^{2}+K \sin ^{2} \theta-J_{\mathrm{int}} \cos \theta-H M \cos \theta\right. \\
& \left.+2 \pi M^{2} \sin ^{2} \theta \sin ^{2} \varphi\right] \mathrm{d} y .
\end{aligned}
$$

In Eq. (1), $\theta(y)$ is the angle between the magnetization and the easy axis of the ferromagnetic film, $z$-axis, which is in the plane of the film, parallel to the uniaxial axis of the antiferromagnetic substrate and to the step edge. $\varphi$ is the out-of-plane angle whose equilibrium value is zero for a Nèel wall. The magnetic field, $H$, is applied along the step edge and $K$ is the effective uniaxial anisotropy constant. The nucleation of a Nèel wall originates from the discontinuous change of direction of the interface exchange field at the step edge, represented by a change of sign of $J_{\text {int }}$ at $y=0$.

The magnetic profile of the domain wall is taken to be of the form

$\tan \frac{\theta(y)}{2}=\exp \left(\frac{y-q_{H}}{\Delta(t)}\right)$

where

$\Delta(t)=\Delta_{0}+\delta \Delta \mathrm{e}^{\mathrm{i} \Omega t}$,

$\Delta_{0}$ and $q_{H}$ are the equilibrium values of the domain wall width and the equilibrium position of the domain wall center, and are determined by energy minimization.

Minimizing the magnetic energy, we get:

$q_{H}=\Delta \tanh ^{-1} \frac{H}{H_{J}}$

and

$\frac{\Delta}{\Delta_{0}}=\frac{1}{\sqrt{1+2\left(H_{J} / H_{A}\right) \ln 2+g(H)}}$.

The field-induced changes of the domain wall width originates from the displacement of the domain wall from the pinning center and is governed by the function $g(H)$ which is given by

$$
\begin{aligned}
g(H)= & \frac{H-H_{J}}{H_{A}} \ln \left(1-\frac{H}{H_{J}}\right) \\
& -\frac{H+H_{J}}{H_{A}} \ln \left(1+\frac{H}{H_{J}}\right) .
\end{aligned}
$$

In the above equations we used $\Delta_{0}=\sqrt{(A / K)}$, $H_{J}=J_{\text {int }} / M$ and $H_{A}=2 \mathrm{~K} / \mathrm{M}$. For $H=0$, from Eq. (5) we have

$$
\frac{\Delta}{\Delta_{0}}=\frac{1}{\sqrt{1+2\left(H_{J} / H_{A}\right) \ln 2}} .
$$

From Eq. (5), it is clear that $\Delta$ is a function of the applied field $H$ and that for materials of low anisotropy, one may find a significant reduction of the domain wall as a result of the step defect of the AF substrate. The maximum reduction occurs for $H=0$, when $\Delta$ has the value given by Eq. (7). When the field increases the effect of the interface defect is made weaker and in the limit of $H=H_{J}$ the intrinsic domain wall width is restored: $\Delta=\Delta_{0}$.

The fluctuations of the domain wall width around the equilibrium value, as given by Eq. (3), leads to fluctuations of the out-of-plane angle, whose equilibrium value for a Nèel wall is zero. We consider

$\varphi(t)=\frac{y-q H}{\Delta_{0}} \phi(t)$

where

$\phi(t)=\phi \mathrm{e}^{\mathrm{i} \Omega t}$.

Notice from Eqs. (8) and (9) that the center of the wall is at rest. The nature of the domain wall mode and the Landau-Lifshitz equations of motion motivate this choice.

In order to define the dynamics in terms of global variables, which describe the breathing motion of the domain wall, we calculate the energy variations imposed by the fluctuations in $\theta$ and $\phi$ and integrate throughout the domain wall.

The global equations of motion for the breathing domain wall, in terms of the dynamical variables $\delta \Delta$ and $\phi$ are

$\frac{\partial \sigma}{\partial \delta \Delta}=-\frac{\pi^{2}}{6} \frac{M}{\gamma} \dot{\phi}$

$\frac{\partial \sigma}{\partial \phi}=-\frac{\pi^{2}}{6} \frac{M}{\gamma} \delta \dot{\Delta}$,

where $\sigma=E / 4 \sqrt{(A K)}$ and the limit of $L \gg \Delta_{0}$ has been used.

The energy, given by Eq. (1), is a quadratic function of $\delta \Delta$ and $\phi$. Using the magnetic profile given by Eqs. (2) and (3), and the values of the domain wall position and the domain wall width given by Eqs. (4) and (5), the frequency of the breathing domain wall mode is obtained.

Considering $4 \pi M \gg H_{A}$, the frequency of the breathing domain wall mode is given by

$\frac{\Omega}{\Omega_{0}}=\sqrt{\frac{12}{\pi^{2}}\left\{\left(\frac{\Delta_{0}}{\Delta}\right)^{2}+\frac{H_{J}}{H_{A}}\left(\frac{q_{H}}{\Delta}\right)^{2}\left(1-\left(\frac{H}{H_{J}}\right)^{2}\right)\right\}}$,

where $\Omega_{0}=\gamma \sqrt{4 \pi M H_{A}}$. 


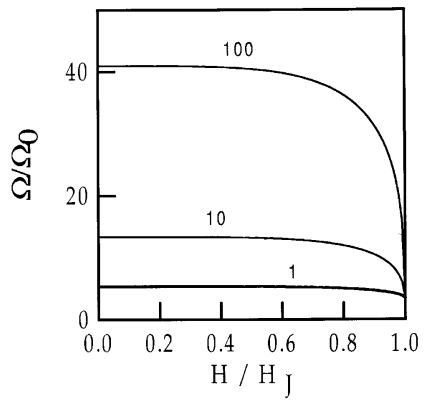

Fig. 1. Breathing domain wall mode frequency. The numbers by the curves indicate the values of $H_{J} / H_{A}$.

In Fig. 1 we display the frequency of the BDWM as a function of the external field. For $H=0$ the interface reduction in the domain wall width is maximum. When the field approaches the interface field value $H=H_{J}$, then the domain wall is far away from the step defect and the domain wall width is restored to its intrinsic value $\Delta_{0}$. In this limit, the BDWM frequency is equal to the value of a free wall, not subjected to the interface step defect. From Eq. (12) we get the value of the intrinsic breathing domain wall mode frequency, $\Omega_{i}$, as

$\Omega_{i}=\Omega_{0} \frac{\sqrt{12}}{\pi}$.

Using Eqs. (7) and (12), we get the frequency for $H=0$, $\Omega(0)$, given by

$\frac{\Omega(0)}{\Omega_{i}}=\sqrt{1+2 \frac{H_{J}}{H_{A}} \ln 2}$
The ratio between the frequency for $H=0$ and the intrinsic value of the breathing domain wall frequency is the ratio between the intrinsic domain wall width and the interface reduced domain wall width. Thus, if the reduction of the domain wall width imposed by the interface coupling is large, the spectrum of breathing domain wall modes is wide. In Fig. 1, a few values of $H_{J} / H_{A}$ have been selected in order to display this feature.

This research was partially supported by the CNPq.

\section{References}

[1] Y. Liu, P. Grutter, J. Appl. Phys. 83 (1998) 7333.

[2] D.T. Pierce, J. Unguris, R.J. Celotta, M.D. Stiles, J. Magn. Magn. Mater. 200 (1999) 290.

[3] A.P. Malozemoff, Phys. Rev. B 35 (1987) 3679.

[4] A.P. Malozemoff, J. Appl. Phys. 63 (1988) 3874.

[5] J. Unguris, R.J. Celotta, D.T. Pierce, Phys. Rev. Lett. 67 (1991) 140.

[6] J. Unguris, R.J. Celotta, D.T. Pierce, Phys. Rev. Lett. 69 (1992) 1125.

[7] E.J. Escorcia-Aparicio, H.J. Choi, W.L. Ling, R.K. Kawakami, Z.Q. Qiu, Phys. Rev. Lett. 81 (1998) 2144.

[8] E.J. Escorcia-Aparicio, J.H. Wolfe, H.J. Choi, W.L. Ling, R.K. Kawakami, Z.Q. Qiu, Phys. Rev. B 59 (1999) 11892.

[9] A.L. Dantas, A.S. Carriço, IEEE Trans. Magn. (2000).

[10] A.L. Dantas, A.S. Carriço, Mat. Sci. Forum 302-303 (1999) 101.

[11] A.L. Dantas, A.S. Carriço, J. Phys.: Condens. Matter 11 (1999) 2707. 